\title{
Dephasing representation of quantum fidelity for general pure and mixed states
}

\author{
Jiří Vaníček* \\ Kenneth S. Pitzer Center for Theoretical Chemistry, University of California, Berkeley, California 94720, USA \\ and Institute for Advanced Study, Princeton, New Jersey 08540, USA
}

(Received 19 May 2005; revised manuscript received 31 December 2005; published 14 April 2006)

\begin{abstract}
A general semiclassical expression for quantum fidelity (Loschmidt echo) of arbitrary pure and mixed states is derived. It expresses fidelity as an interference sum of dephasing trajectories weighed by the Wigner function of the initial state, and does not require that the initial state be localized in position or momentum. This general dephasing representation is special in that, counterintuitively, all of fidelity decay is due to dephasing and none is due to the decay of classical overlaps. Surprising accuracy of the approximation is justified by invoking the shadowing theorem: twice-both for physical perturbations and for numerical errors. Beyond justifying the approximation, the shadowing theorem makes the dephasing representation practical: without shadowing it would be impossible to find numerically the precise trajectories needed in a semiclassical approximation. It is shown how the general expression reduces to the previously known special forms for localized states. The superiority of the general over the specialized forms is explained and supported by numerical tests for wave packets, nonlocal pure states, and for simple and random mixed states. The tests are done in nonuniversal regimes in mixed phase space where detailed features of fidelity are important. Although semiclassically motivated, the present approach is valid for abstract systems with a finite Hilbert basis provided that the discrete Wigner transform is used. This makes the method applicable, via a phase-space approach, to problems of quantum computation.
\end{abstract}

DOI: 10.1103/PhysRevE.73.046204

PACS number(s): 05.45.Mt, 03.65.Sq, 02.70.-c

\section{INTRODUCTION}

Time evolution in classical mechanics is very sensitive to perturbations of both initial conditions of a trajectory and the Hamiltonian. Because of the unitarity of quantum evolution, on the other hand, the overlap of two different quantum states remains constant in time. However, we can still define sensitivity of quantum evolution to perturbations of the Hamiltonian. This is usually done using the notion of quantum fidelity (sometimes called Loschmidt echo), defined for pure states as [1]

$$
\begin{gathered}
M(t)=|O(t)|^{2}, \\
O(t)=\left\langle\psi\left|e^{+i H^{\epsilon} t / \hbar} e^{-i H^{0} t / \hbar}\right| \psi\right\rangle,
\end{gathered}
$$

where $O(t)$ is the fidelity amplitude, $|\psi\rangle\rangle$ is the initial state, and $H^{0}$ and $H^{\epsilon}=H^{0}+\epsilon V$ are the unperturbed and perturbed Hamiltonians, respectively. In words, fidelity is the squared overlap at time $t$ of two identical initial states evolved with two slightly different Hamiltonians. Because of its relevance in theories of decoherence and in experimental realizations of quantum computation [2], quantum as well as classical fidelity has been extensively studied in the last few years; for a partial review, see Ref. [3]. One of the main results of the intensive study of fidelity is the identification of various universal regimes of fidelity decay in different limiting cases [4-16]. The connection between fidelity decay and decoherence is discussed in Refs. [17-19]. Several experiments that measure fidelity (or fidelity amplitude) also exist [20-22].

\footnotetext{
*Electronic address: vanicek@ post.harvard.edu
}

Many of the theoretical works used a semiclassical (SC) approach, but before Ref. [23] only as a starting point for various approximations, because of difficulties in treating an exponentially growing number of terms in the general SC expression for fidelity, especially in chaotic systems. This problem was solved in Ref. [23] by a uniform expression for fidelity amplitude which implicitly summed over all these contributions using an integral over initial conditions, similar in spirit to Miller's initial value representation [24,25]. This surprisingly simple and accurate expression, although limited to wave packets localized in position, has been successfully applied as a starting point to derive fidelity decay in the deep Lyapunov regime [26] and the plateau of fidelity in neutron scattering [16].

In a recent paper [27], the uniform expression for fidelity was justified by the shadowing theorem of classical mechanics $[28,29]$ and a more general and, in fact, always more accurate expression,

$$
O_{\mathrm{DR}}(t)=\int d \mathbf{r}_{0} \int d \mathbf{p}_{0} \rho_{W}\left(\mathbf{r}_{0}, \mathbf{p}_{0}\right) \exp \left[-i \Delta S_{t}^{\epsilon}\left(\mathbf{r}_{0}, \mathbf{p}_{0}\right) / \hbar\right],
$$

valid for arbitrary pure states, was stated. In this dephasing representation (DR), fidelity amplitude is expressed as an interference integral over "dephasing trajectories" with initial conditions $\mathbf{r}_{0}, \mathbf{p}_{0}$ and weighed by the Wigner function $\rho_{W}$ of the initial state. The phase is given by the integral $\Delta S_{t}^{\epsilon}=$ $-\epsilon \int_{0}^{t} d \tau V\left(\mathbf{r}_{\tau}, \tau\right)$ of the perturbation along the unperturbed trajectory. One purpose of the present paper is to provide (in Sec. II) a detailed derivation of this general DR for arbitrary pure, i.e., also nonlocal states. The DR is derived by applying the shadowing theorem to approximate the stationary- 
phase points of a more general uniform SC expression. Shadowing is central to the DR for two main reasons: the shadowing theorem justifies the approximation theoretically as well as makes it practical numerically. Previously, the shadowing theorem has most often been used to justify numerical simulations of classical quantities in chaotic systems. It does so by considering ensemble averages and guaranteeing the existence of an exact trajectory with a slightly displaced initial condition that "shadows" a numerically noisy trajectory. Dephasing representation offers a completely different and perhaps even more surprising application of the theorem: shadowing is used to compute a quantummechanical rather than a classical quantity. Moreover, the theorem is applied primarily to physical rather than numerical perturbations.

In Sec. III it is shown that the same DR is valid also for general mixed states. Section IV shows how the general expression reduces to the original form [23] and other specialized forms for position and momentum states or Gaussian wave packets localized in position or momentum. In Sec. V, the general DR is tested on a nonlocal state-a coherent superposition of two position states-and on two types of mixed state-an incoherent superposition of two position states and a random mixture of pure states. It is also shown that the general expression is superior to the original form [23] even for a single Gaussian wave packet. All numerical calculations are done for a system with a finite Hilbert basis. In such systems, quantum phase space can be rigorously defined if the original Wigner function [30] is replaced by the discrete Wigner transform [31-33]. Since this discrete transform can be defined in a general abstract Hilbert space with finite basis, the present approach should be applicable to problems of quantum computation if phase space approach is used [34]. Numerical errors of the DR are analyzed in Sec. VI. In Sec. VII, DR is compared to other "Wigner" methods. The main conclusions of the paper are summarized in Sec. VIII.

\section{DEPHASING REPRESENTATION FOR A GENERAL PURE STATE}

Fidelity amplitude for a general pure state $|\psi\rangle$ is given by Eq. (2). In order to derive the general dephasing representation (3) of fidelity, we could start by replacing the two quantum propagators in Eq. (2) by the corresponding SC Van Vleck propagators [35], as in Refs. [4,23,27]. However, we will save some effort if we start directly from the SC initial value representation (IVR) [24,25] for the Van Vleck propagator,

$$
e^{-i H^{\epsilon} t / \hbar} \approx(2 \pi i \hbar)^{-d / 2} \int d \mathbf{x}_{0}^{\epsilon}\left|\partial \mathbf{r}_{t}^{\epsilon}\left(\mathbf{x}_{0}^{\epsilon}\right) / \partial \mathbf{p}_{0}^{\epsilon}\right|^{1 / 2} e^{i S_{t}^{\epsilon}\left(\mathbf{x}_{0}^{\epsilon}\right) / \hbar}\left|\mathbf{r}_{t}^{\epsilon}\right\rangle\left\langle\mathbf{r}_{0}^{\epsilon}\right| .
$$

Here $\mathbf{x}=(\mathbf{r}, \mathbf{p})$ denotes the phase space point with position $\mathbf{r}$ and momentum $\mathbf{p}$, the subscript $(t)$ is the time of propagation, and the superscript $(\epsilon)$ is the value of the perturbation used for propagation. E.g., $\mathbf{x}_{0}^{0}$ denotes the initial conditions of trajectories of $H^{0}$. Action $S_{t}^{\epsilon}$ of a trajectory of the Hamiltonian $H^{\epsilon}$ is given by

$$
S_{t}^{\epsilon}\left(\mathbf{x}_{0}^{\epsilon}\right)=\int_{0}^{t} d \tau\left[\mathbf{p}_{\tau}^{\epsilon} \cdot \dot{\mathbf{r}}_{\tau}^{\epsilon}-H^{\epsilon}\left(\mathbf{x}_{\tau}^{\epsilon} ; \tau\right)\right]
$$

In the simplified notation above, the square roots of the determinants in Eq. (4) also include the appropriate Maslov indices [36]. Using the IVR expressions (4), fidelity amplitude (2) becomes

$$
\begin{aligned}
O_{\mathrm{IVR}}(t)= & (2 \pi \hbar)^{-d} \int d \mathbf{x}_{0}^{0} \int d \mathbf{x}_{0}^{\epsilon}\left|\frac{\partial \mathbf{r}_{t}^{0}}{\partial \mathbf{p}_{0}^{0}}\right|^{1 / 2}\left|\frac{\partial \mathbf{r}_{t}^{\epsilon}}{\partial \mathbf{p}_{0}^{\epsilon}}\right|^{1 / 2}\left\langle\psi \mid \mathbf{r}_{0}^{\epsilon}\right\rangle \\
& \times\left\langle\mathbf{r}_{t}^{\epsilon} \mid \mathbf{r}_{t}^{0}\right\rangle\left\langle\mathbf{r}_{0}^{0} \mid \psi\right\rangle e^{i\left(S_{t}^{0}-S_{t}^{\epsilon}\right) / \hbar}
\end{aligned}
$$

\section{A. Uniform semiclassical expression for fidelity}

If we further expand the overlap $\left\langle\mathbf{r}_{t}^{\epsilon} \mid \mathbf{r}_{t}^{0}\right\rangle$ in integral (6) as an integral over a dummy momentum $\mathbf{q}$,

$$
\left\langle\mathbf{r}_{t}^{\epsilon} \mid \mathbf{r}_{t}^{0}\right\rangle=\delta\left(\Delta \mathbf{r}_{t}\right)=(2 \pi \hbar)^{-d} \int d \mathbf{q} e^{i \mathbf{q} \cdot \Delta \mathbf{r}_{t} / \hbar},
$$

with $\Delta \mathbf{r}_{t}=\mathbf{r}_{t}^{\epsilon}-\mathbf{r}_{t}^{0}$, we obtain a "full" uniform SC expression for fidelity,

$$
\begin{aligned}
O_{\text {unif }}(t)= & (2 \pi \hbar)^{-2 d} \int d \mathbf{x}_{0}^{0} \int d \mathbf{x}_{0}^{\epsilon} \int d \mathbf{q}\left|\frac{\partial \mathbf{r}_{t}^{0}}{\partial \mathbf{p}_{0}^{0}}\right|^{1 / 2}\left|\frac{\partial \mathbf{r}_{t}^{\epsilon}}{\partial \mathbf{p}_{0}^{\epsilon}}\right|^{1 / 2} \\
& \times \psi^{*}\left(\mathbf{r}_{0}^{\epsilon}\right) \psi\left(\mathbf{r}_{0}^{0}\right) \times \exp \left[i\left(S_{t}^{0}-S_{t}^{\epsilon}+\mathbf{q} \cdot \Delta \mathbf{r}_{t}\right) / \hbar\right]
\end{aligned}
$$

This integral is, formally, semiclassically "exact." In particular, it is free of caustics, unlike, e.g., the Van Vleck propagator. Also, it includes the "off-diagonal" terms that automatically disappear in the dephasing representation (3). In this sense, expression (7) is the ultimate SC representation of fidelity amplitude. Because $O_{\text {unif }}$ is expressed only in terms of initial conditions (and dummy momentum q), it appears to be ready for numerical evaluations. Unfortunately, this integral is highly oscillatory, and very difficult to compute, especially in many-dimensional or chaotic systems. Therefore we will take an alternative route, using a further approximation, but obtain an integral (3) much easier to tackle numerically and which appears to give much better numerical results than Eq. (7). Nevertheless, uniform expression (7) deserves a more detailed examination in the future, in particular since it could shed light on the controversy surrounding the so-called diagonal approximation. This is, however, beyond the scope of the present paper.

\section{B. Dephasing representation}

Before making any further approximations, let us perform a change of variables $\left\{\mathbf{x}^{0}, \mathbf{x}^{\epsilon}\right\} \rightarrow\{\mathbf{x}, \Delta \mathbf{x}\}$ in integral (6). It should be emphasized that we do not assume $\Delta \mathbf{x}$ to be small. New variables (averages and differences) are defined for all times from 0 to $t$ as

$$
\begin{gathered}
\mathbf{x}=\frac{1}{2}\left(\mathbf{x}^{0}+\mathbf{x}^{\epsilon}\right), \\
\Delta \mathbf{x}=\mathbf{x}^{\epsilon}-\mathbf{x}^{0} .
\end{gathered}
$$

The Jacobian of this transformation is unity, so 


$$
\begin{aligned}
O(t)= & (2 \pi \hbar)^{-d} \int d \mathbf{x}_{0} \int d \Delta \mathbf{x}_{0}\left|\frac{\partial \mathbf{r}_{t}^{0}}{\partial \mathbf{p}_{0}^{0}}\right|^{1 / 2}\left|\frac{\partial \mathbf{r}_{t}^{\epsilon}}{\partial \mathbf{p}_{0}^{\epsilon}}\right|^{1 / 2} \\
& \times \psi^{*}\left(\mathbf{r}_{0}^{\epsilon}\right) \delta\left(\Delta \mathbf{r}_{t}\right) \psi\left(\mathbf{r}_{0}^{0}\right) \exp \left(\frac{i}{\hbar}\left(S_{t}^{0}-S_{t}^{\epsilon}\right)\right) .
\end{aligned}
$$

While we do not intend to evaluate this integral fully by the stationary phase (SP) approximation, it is instructive to check where the action difference $S_{t}^{0}-S_{t}^{\epsilon}$ is stationary because those regions give the main contributions to the integral. Variation of action $S_{t}^{\epsilon}$ gives

$$
\delta S_{t}^{\epsilon}=-\mathbf{p}_{0}^{\epsilon} \cdot \delta \mathbf{r}_{0}^{\epsilon}+\mathbf{p}_{t}^{\epsilon} \cdot \delta \mathbf{r}_{t}^{\epsilon} .
$$

Variation of the action difference $S_{t}^{0}-S_{t}^{\epsilon}$ in terms of the variations of variables $\{\mathbf{r}, \Delta \mathbf{r}\}$ becomes

$$
\delta\left(S_{t}^{0}-S_{t}^{\epsilon}\right)=\Delta \mathbf{p}_{0} \cdot \delta \mathbf{r}_{0}+\mathbf{p}_{0} \cdot \delta \Delta \mathbf{r}_{0}-\Delta \mathbf{p}_{t} \cdot \delta \mathbf{r}_{t}-\mathbf{p}_{t} \cdot \delta \Delta \mathbf{r}_{t} .
$$

Due to the $\Delta \mathbf{r}_{t}=0$ constraint, we have a constraint $\delta \Delta \mathbf{r}_{t}$ $=0$ on the variation of endpoints. Expanding variations of positions at time $t$ in terms of variations of phase-space coordinates at time 0 , we find

$$
\begin{aligned}
\delta\left(S_{t}^{0}-S_{t}^{\epsilon}\right)= & \left(\Delta \mathbf{p}_{0}-\Delta \mathbf{p}_{t} \cdot \frac{\partial \mathbf{r}_{t}}{\partial \mathbf{r}_{0}}\right) \cdot \delta \mathbf{r}_{0}-\Delta \mathbf{p}_{t} \cdot \frac{\partial \mathbf{r}_{t}}{\partial \mathbf{r}_{0}} \cdot \delta \mathbf{p}_{0} \\
& +\mathbf{p}_{0} \cdot \delta \Delta \mathbf{r}_{0} .
\end{aligned}
$$

There are three stationary phase conditions,

$$
\begin{gathered}
\Delta \mathbf{p}_{0}-\Delta \mathbf{p}_{t} \cdot \frac{\partial \mathbf{r}_{t}}{\partial \mathbf{r}_{0}}=0, \\
\Delta \mathbf{p}_{t} \cdot \frac{\partial \mathbf{r}_{t}}{\partial \mathbf{r}_{0}}=0, \\
\mathbf{p}_{0} \cdot \delta \Delta \mathbf{r}_{0}=0 .
\end{gathered}
$$

The third SP condition was intentionally written in the full form. The solution to these three equations is

$$
\Delta \mathbf{p}_{0}=\Delta \mathbf{p}_{t}=\Delta \mathbf{r}_{0}=0
$$

In general, all three conditions would be satisfied only for a discrete set of trajectories (together with the constraint $\Delta \mathbf{r}_{t}$ $=\mathbf{0}$ there are $4 d$ equations for $4 d$ unknowns $\left.\mathbf{x}_{0}, \Delta \mathbf{x}_{0}\right)$. However, for zero perturbation $(\epsilon=0)$, one immediately sees that the solution (14) exists for each pair $\left(\mathbf{r}_{0}, \mathbf{p}_{0}\right)$ : the solution consists of identical trajectories $\Delta \mathbf{x}_{\tau}=\mathbf{0}$ for all times $\tau, 0$ $<\tau<t$. As we shall see below in Eq. (23), also the final result for fidelity becomes exact in this limit $(\epsilon=0)$. If we add the perturbation, these precise solutions break down, due to the exponential sensitivity of classical dynamics. Due to the exponential divergence of trajectories with $\Delta \mathbf{x}_{0}=0$, we would only expect a discrete number of fortuitous solutions satisfying $\Delta \mathbf{x}_{t}=0$. However, as was shown in Ref. [27], if the shadowing theorem $[28,29]$ is applicable in the given system (for a given perturbation $\epsilon$ and up to time $t$ ), there will be a very near solution with $\Delta \mathbf{x}_{\tau} \approx \mathbf{0}$ for all times $\tau, 0<\tau<t$. Putting off a discussion of the shadowing theorem until later, suffice it to say that this theorem, completely counterintuitively, guarantees that we can compensate one exponential sensitivity (to perturbations of $H^{0}$ ) by another exponential sensitivity (to initial conditions) and get a trajectory which remains very close to the unperturbed trajectory up to time $t$. Assuming the validity of shadowing, the solutions dephase as

$$
\begin{gathered}
S_{t}^{0}-S_{t}^{\epsilon} \approx-\Delta S_{t}^{\epsilon}\left(\mathbf{x}_{0}\right)-\Delta \mathbf{r}_{t} \cdot \mathbf{p}_{t}+\Delta \mathbf{r}_{0} \cdot \mathbf{p}_{0} \\
\Delta S_{t}^{\epsilon}\left(\mathbf{x}_{0}\right)=-\epsilon \int_{0}^{t} d \tau V\left(\mathbf{r}_{\tau}, \tau\right) .
\end{gathered}
$$

The first term in Eq.(15), $\Delta S_{t}\left(\mathbf{x}_{0}\right)$, is due to the perturbing potential $\epsilon V$ along the unperturbed trajectory, the other two terms are due to the small difference of trajectories at time $t$ and at time 0 . We shall use the action difference (15) to simplify integral (9) for fidelity amplitude. If we plan to evaluate the integral over $\Delta \mathbf{x}_{0}$ first, we can assume $\mathbf{x}_{0}$ fixed for a moment. Then for small $\Delta \mathbf{x}_{0}$, we can approximate the product of the two Jacobians in Eq. (9) as

$$
\begin{aligned}
\left|\frac{\partial \mathbf{r}_{t}^{0}}{\partial \mathbf{p}_{0}^{0}}\right|^{1 / 2}\left|\frac{\partial \mathbf{r}_{t}^{\epsilon}}{\partial \mathbf{p}_{0}^{\epsilon}}\right|^{1 / 2} & \approx\left|\frac{\partial\left(-\Delta \mathbf{r}_{t}\right)}{\partial\left(-\Delta \mathbf{p}_{0}\right)}\right|^{1 / 2}\left|\frac{\partial \Delta \mathbf{r}_{t}}{\partial \Delta \mathbf{p}_{0}}\right|^{1 / 2} \\
& =\left|\frac{\partial \Delta \mathbf{r}_{t}}{\partial \Delta \mathbf{p}_{0}}\right|\left(\mathbf{x}_{0}\right)
\end{aligned}
$$

and change variables from $\Delta \mathbf{p}_{0}$ to $\Delta \mathbf{r}_{t}$ in Eq. (9),

$$
\begin{aligned}
O(t)= & (2 \pi \hbar)^{-d} \int d \mathbf{x}_{0} \int d \Delta \mathbf{r}_{0} \int d \Delta \mathbf{r}_{t} \psi^{*}\left(\mathbf{r}_{0}^{\epsilon}\right) \delta\left(\Delta \mathbf{r}_{t}\right) \\
& \times \psi\left(\mathbf{r}_{0}^{0}\right) \exp \left(\frac{i}{\hbar}\left(S_{t}^{0}-S_{t}^{\epsilon}\right)\right) .
\end{aligned}
$$

Integration over $\Delta \mathbf{r}_{t}$ eliminates the $\delta$ function,

$$
\begin{aligned}
O(t)= & (2 \pi \hbar)^{-d} \int d \mathbf{x}_{0} \int d \Delta \mathbf{r}_{0} \psi^{*}\left(\mathbf{r}_{0}^{\epsilon}\right) \psi\left(\mathbf{r}_{0}^{0}\right) \\
& \times\left.\exp \left(\frac{i}{\hbar}\left(S_{t}^{0}-S_{t}^{\epsilon}\right)\right)\right|_{\Delta \mathbf{r}_{t}=0} \cdot
\end{aligned}
$$

Substituting the action difference (15) into integral (19), we obtain our main result, the dephasing representation (3),

$$
\begin{aligned}
O_{\mathrm{DR}}(t)= & (2 \pi \hbar)^{-d} \int d \mathbf{x}_{0} \int d \Delta \mathbf{r}_{0} \psi^{*}\left(\mathbf{r}_{0}+\frac{1}{2} \Delta \mathbf{r}_{0}\right) \\
& \times \psi\left(\mathbf{r}_{0}-\frac{1}{2} \Delta \mathbf{r}_{0}\right) \exp \left(\frac{i}{\hbar}\left(-\Delta S_{t}^{\epsilon}+\Delta \mathbf{r}_{0} \cdot \mathbf{p}_{0}\right)\right)
\end{aligned}
$$

The final result is more succinctly written as

$$
O_{\mathrm{DR}}(t)=\int d \mathbf{x}_{0} \rho_{W}\left(\mathbf{x}_{0}\right) \exp \left[-i \Delta S_{t}^{\epsilon}\left(\mathbf{x}_{0}\right) / \hbar\right]
$$

using the Wigner function of the initial state $|\psi\rangle$, 


$$
\begin{aligned}
\rho_{W}(\mathbf{r}, \mathbf{p})= & (2 \pi \hbar)^{-d} \int d \Delta \mathbf{r} \psi^{*}\left(\mathbf{r}+\frac{1}{2} \Delta \mathbf{r}\right) \\
& \times \psi\left(\mathbf{r}-\frac{1}{2} \Delta \mathbf{r}\right) \exp (i \Delta \mathbf{r} \cdot \mathbf{p} / \hbar) .
\end{aligned}
$$

The general expression (21) expresses fidelity as an interference integral over initial positions $\mathbf{r}_{0}$ and momenta $\mathbf{p}_{0}$. The amplitude of each term is given by the Wigner function $\rho_{W}\left(\mathbf{r}_{0}, \mathbf{p}_{0}\right)$ and the phase by the integral of the perturbing potential along the unperturbed trajectory, $\Delta S_{t}^{\epsilon}\left(\mathbf{r}_{0}, \mathbf{p}_{0}\right)$. As time increases, each trajectory accumulates phase due to the perturbing potential and its contribution "dephases" in comparison with the situation where perturbation vanishes. In Eq. (21), decay of fidelity appears - surprisingly-to be completely due to the destructive interference (caused by this dephasing) and not at all due to the decay of classical overlaps (caused by the perturbation of classical trajectories). Nevertheless, as will be seen in the numerical examples below, Eq. (21) captures even the component of fidelity decay that is due to the decay of classical overlaps in the usual SC picture. Because of these surprising properties, expression (21) was called dephasing representation in Ref. [27].

Although we started our derivation for a pure state, we ended up with a DR in terms of the Wigner function. Since this function can also be defined for mixed states, it appears that expression (21) should remain valid for mixed states, with appropriate generalization of the notion of fidelity. In Sec. III, it will be shown that this is indeed the case.

It should be pointed out that a separate diagonal approximation was not necessary in this approach: the majority of nearly stationary-phase solutions are nearly "diagonal" $\left(\Delta \mathbf{x}_{\tau} \approx 0\right)$. Whereas the solutions $\Delta \mathbf{x}_{0} \approx 0$ exist for every $\mathbf{x}_{0}$ ( $2 d$-dimensional solution space), other solutions are very special and exist only for discrete values of $\mathbf{x}_{0}$ and $\Delta \mathbf{x}_{0}$ (0-dimensional solution space). In other SC approaches (which do not use an initial value representation), a separate diagonal approximation is necessary and is justified by averaging over impurities or over initial states [4,7]. However, in certain situations, using a periodic orbit expansion rather than an IVR integral over the whole phase space, offdiagonal solutions have been shown to be important $[37,38]$ for long times where almost degenerate periodic orbits exist. It would be interesting to see if there are systems or regimes in which the DR severely breaks down because of the offdiagonal terms. The only breakdown of DR of which the author is aware is in the perturbative regime after the Heisenberg time [23]. But in general, a SC approximation that does not consider the discreteness of the spectrum is not expected to work after the Heisenberg time. The computational advantage of the DR over other SC methods in general is that the search for periodic orbits or other special classical trajectories is not necessary. If off-diagonal terms of the type that Sieber and Richter [37] discuss were important, it would be difficult to include them in the dephasing approximation explicitly without losing its efficiency. On the other hand, the off-diagonal terms are implicitly included in the uniform method (7), which is unfortunately computationally much more expensive if at all tractable, especially in higherdimensional systems.
There is another simple argument in favor of the DR and against considering other terms than those with $\Delta \mathbf{x}_{\tau} \approx 0$. For zero perturbations, $\epsilon=0$, expression (21) correctly reduces to the exact result,

$$
O_{\mathrm{DR}}^{\epsilon=0}(t)=\int d \mathbf{x}_{0} \rho_{W}\left(\mathbf{x}_{0}\right)=1,
$$

for all times $t$, where the basic property of the Wigner function was used. This simple result is reassuring because the "more accurate" SC expressions such as the uniform result (7) or the nonuniform Van Vleck representations from Refs. $[4,7]$ (before the diagonal approximation) do not reduce to the exact result for zero perturbations. Including the "offdiagonal" terms in this case would break equality (23).

\section{Shadowing theorem and its double use}

Shadowing theorems in general state that (under certain detailed conditions) for small enough $\epsilon$ there is a time $t$ such that for a trajectory of $H^{0}$ with initial condition $\mathbf{r}_{0}^{0}, \mathbf{p}_{0}^{0}$ there exists a trajectory of $H^{\epsilon}$ with initial condition $\mathbf{r}_{0}^{\epsilon}, \mathbf{p}_{0}^{\epsilon}$ remaining within a certain small distance from the first trajectory up to time $t$. In uniformly hyperbolic systems this shadowing time $t$ is infinite $[39,40]$, in more general systems at least finite [29]. Since it is very difficult to find the maximum shadowing time $t$ and the corresponding bound on the closeness of trajectories for a specific system, the derivation of DR of fidelity assumed that shadowing was applicable for a given perturbation and time: the numerical results will provide the final verification.

In order to use DR in numerical applications, one only needs to generate initial conditions $\mathbf{r}_{0}, \mathbf{p}_{0}$ from a distribution given by the Wigner function $\rho_{W}$, run trajectories with the unperturbed Hamiltonian $H^{0}$ and compute the action difference $\Delta S_{t}^{\epsilon}=-\epsilon \int_{0}^{t} d \tau V\left(\mathbf{r}_{\tau}, \tau\right)$ along this trajectory. There is no need to compute Van Vleck determinants or Maslov indices as in many other SC applications. Because the Wigner function, unlike classical probability, can be negative, some care must be taken to sample from this distribution. The simplest possible recipe would be to sample according to the probability $\left|\rho_{W}\right|$ and attach a sign afterward together with the dephasing factor. As we will see from the analysis of special cases in Sec. IV, Wigner function is particularly simple for position and momentum eigenstates (just a delta function), for Gaussian wave packets (a Gaussian in both position and momentum), or for a random mixed state (a constant over the whole phase space). These distributions can be easily sampled using standard methods. For general pure or mixed states one can resort to a Monte Carlo procedure, e.g., using the Metropolis algorithm, which is frequently done for the IVR approximation [25].

One might object that numerical computation of trajectories, due to the exponential sensitivity of classical evolution, will destroy the validity of the DR (21). However, here the shadowing theorem helps again-in fact in its original form $[28,29]$ where the perturbation was indeed due to errors of numerical propagation. The shadowing idea, as stated in Refs. [28,29], guarantees that for each numerical (noisy) trajectory there will be a nearby exact trajectory of $H^{0}$. 


\section{DEPHASING REPRESENTATION FOR A GENERAL MIXED STATE}

There are at least two ways to generalize the pure-state definition (2) of fidelity to mixed states. The simplest generalization is in terms of fidelity amplitude,

$$
O_{\text {mixed } 1}(t)=\operatorname{tr}\left(e^{-i H^{0} t / \hbar} \rho e^{+i H^{\epsilon} t / \hbar}\right),
$$

where $\rho$ is the density matrix of the mixed state, normalized such that $\operatorname{tr} \rho=1[11]$. For pure states $\rho=|\psi\rangle\langle\psi|$, this general definition reduces to the pure-state definition (2). One interpretation of the general expression (24) is that the ket vectors evolve with the unperturbed Hamiltonian $H^{0}$ and the bra vectors with the perturbed Hamiltonian $H^{\epsilon}$. Another interpretation is that expression (24) is simply an average of fidelity amplitudes of pure-state components of the given mixed state. This should be distinguished from the often studied averaged fidelity.

The second possible generalization of the notion of fidelity to mixed states replaces the expression for fidelity (1), rather than fidelity amplitude (2), by an expression

$$
M_{\text {mixed } 2}(t)=\operatorname{tr}\left[\rho^{0}(t) \rho^{\epsilon}(t)\right] / \operatorname{tr} \rho^{2}=\operatorname{tr}[\rho(0) \rho(t)] / \operatorname{tr} \rho^{2},
$$

where $\rho^{0}(t), \rho^{\epsilon}(t)$ are the evolved density operators,

$$
\rho^{\epsilon}(t)=e^{-i H^{\epsilon} t / \hbar} \rho e^{+i H^{\epsilon} t / \hbar},
$$

or, alternatively, $\rho(t)$ is the evolved operator,

$$
\rho(t)=e^{+i H^{\epsilon} t / \hbar} e^{-i H^{0} t / \hbar} \rho e^{+i H^{0} t / \hbar} e^{-i H^{\epsilon} t / \hbar} .
$$

Again for pure states $\rho=|\psi\rangle\langle\psi|$, definition (25) reduces to the pure-state definition (1).

In what follows the simplest generalization (24) in terms of fidelity amplitude is assumed. There are three reasons for this choice: First, there is a simple interpretation: fidelity amplitude of a mixed state is the average of fidelity amplitudes of the pure-state components of the given mixed state. Second, the DR (21) directly generalizes to mixed states when this definition is used. Third, there are both experiments [22] and an experimental proposal [41] that measure averaged fidelity amplitude rather than averaged fidelity (that is usually considered in literature) or expression (25).

With the mixed-state definition (24), the SC derivation in Eqs. (4)-(21) can be followed closely for mixed states, if we replace the product $\left\langle\psi \mid \mathbf{r}_{0}^{\epsilon}\right\rangle\left\langle\mathbf{r}_{0}^{0} \mid \psi\right\rangle=\psi^{*}\left(\mathbf{r}_{0}^{\epsilon}\right) \psi\left(\mathbf{r}_{0}^{0}\right)$ in Eqs. (6), (9), (19), and (20) by the matrix element $\left\langle\mathbf{r}_{0}^{0}|\rho| \mathbf{r}_{0}^{\epsilon}\right\rangle$ of the density operator. At the end, we obtain the same final result (21), only the Wigner function of a pure state (22) must be replaced by the Wigner-Weyl transform of the density operator,

$$
\begin{aligned}
\rho_{W}(\mathbf{r}, \mathbf{p})= & (2 \pi \hbar)^{-d} \int d \Delta \mathbf{r}\left\langle\mathbf{r}+\frac{1}{2} \Delta \mathbf{r}|\rho| \mathbf{r}-\frac{1}{2} \Delta \mathbf{r}\right\rangle \\
& \times \exp (i \Delta \mathbf{r} \cdot \mathbf{p} / \hbar) .
\end{aligned}
$$

\section{SPECIAL CASES}

For a position state $|\mathbf{R}\rangle, \psi(\mathbf{r})=\delta(\mathbf{r}-\mathbf{R})$, Wigner function (22) is

$$
\rho_{W}^{\text {pos.st. }}(\mathbf{r}, \mathbf{p})=(2 \pi \hbar)^{-d} \delta(\mathbf{r}-\mathbf{R}) .
$$

Substituting Eq. (22) into the general DR (21), we find

$$
O_{\mathrm{DR}}^{\text {pos.st. }}(t)=(2 \pi \hbar)^{-d} \int d \mathbf{p}_{0} \exp \left[-i \Delta S_{t}^{\epsilon}\left(\mathbf{R}, \mathbf{p}_{0}\right) / \hbar\right]
$$

in agreement with Eq. (1) from Ref. [27] and with Ref. [23].

For a momentum state $|\mathbf{P}\rangle, \psi(\mathbf{r})=(2 \pi \hbar)^{-d / 2} \exp (i \mathbf{P} \cdot \mathbf{r} / \hbar)$, Wigner function (22) becomes

$$
\rho_{W}^{\text {mom.st. }}(\mathbf{r}, \mathbf{p})=(2 \pi \hbar)^{-d} \delta(\mathbf{p}-\mathbf{P})
$$

and the general DR of fidelity (21) reduces to

$$
O_{\mathrm{DR}}^{\text {mom.st. }}(t)=(2 \pi \hbar)^{-d} \int d \mathbf{r}_{0} \exp \left[-i \Delta S_{t}^{\epsilon}\left(\mathbf{r}_{0}, \mathbf{P}\right) / \hbar\right] .
$$

A general Gaussian wave packet with average position $\mathbf{R}$, average momentum $\mathbf{P}$, and position spread $\sigma$,

$$
\psi(\mathbf{r})=\left(\pi \sigma^{2}\right)^{-d / 4} \exp \left[i \mathbf{P} \cdot(\mathbf{r}-\mathbf{R}) / \hbar-(\mathbf{r}-\mathbf{R})^{2} / 2 \sigma^{2}\right]
$$

has Wigner function

$$
\rho_{W}^{\text {gen.G.w.p. }}(\mathbf{r}, \mathbf{p})=(\pi \hbar)^{-d} \exp \left[-(\mathbf{r}-\mathbf{R})^{2} / \sigma^{2}-(\mathbf{p}-\mathbf{P})^{2} \sigma^{2} / \hbar^{2}\right]
$$

In general the DR of a Gaussian wave packet is Eq. (21) with the Wigner function (29) where we must include dephasing trajectories with varying both positions and momenta. Only in special cases, such as when the wave packet is strongly localized in position (i.e., when $\sigma \ll \hbar^{1 / 2}$ ), can we make a further simplification by replacement of $\Delta S_{t}^{\epsilon}\left(\mathbf{r}_{0}, \mathbf{p}_{0}\right)$ by $\Delta S_{t}^{\epsilon}\left(\mathbf{R}, \mathbf{p}_{0}\right)$ in Eq. (21). Then we can compute the $\mathbf{r}_{0}$ integral in Eq. (21) analytically and obtain

$$
\begin{aligned}
O_{\mathrm{DR}}^{\text {pos.G.w.p. }}(t)= & \left(\sigma^{2} / \pi \hbar^{2}\right)^{d / 2} \int d \mathbf{p}_{0} \exp \left[-i \Delta S_{t}^{\epsilon}\left(\mathbf{R}, \mathbf{p}_{0}\right) / \hbar\right. \\
& \left.-(\mathbf{p}-\mathbf{P})^{2} \sigma^{2} / \hbar^{2}\right],
\end{aligned}
$$

in agreement with Eq. (8) in Ref. [23]. There the same result was obtained by linearizing the Van Vleck SC propagator about the central trajectory. In Sec. V it will be shown that the symmetric expression (29) based on the general DR (21) is superior to the specialized form (30). Similarly, if the initial Gaussian wave packet is localized in momentum (i.e., when $\left.\sigma \gg \hbar^{1 / 2}\right)$, we can replace $\Delta S_{t}^{\epsilon}\left(\mathbf{r}_{0}, \mathbf{p}_{0}\right)$ by $\Delta S_{t}^{\epsilon}\left(\mathbf{r}_{0}, \mathbf{P}\right)$ and obtain

$$
\begin{aligned}
O_{\mathrm{DR}}^{\text {mom.G.w.p. }}(t)= & \left(\pi \sigma^{2}\right)^{-d / 2} \int d \mathbf{r}_{0} \exp \left[-i \Delta S_{t}^{\epsilon}\left(\mathbf{r}_{0}, \mathbf{P}\right) / \hbar\right. \\
& \left.-(\mathbf{r}-\mathbf{R})^{2} / \sigma^{2}\right] .
\end{aligned}
$$

For general (non-Gaussian) wave packets, which are nevertheless localized either in position (about $\mathbf{R}$ ) or momentum 
(about $\mathbf{P}$ ), we can use the general property of the Wigner function

$$
\begin{gathered}
\int d \mathbf{r} \rho_{W}(\mathbf{r}, \mathbf{p})=|\psi(\mathbf{p})|^{2}, \\
\int d \mathbf{p} \rho_{W}(\mathbf{r}, \mathbf{p})=|\psi(\mathbf{r})|^{2},
\end{gathered}
$$

and obtain, upon substitution into the general DR (21),

$$
\begin{gathered}
O_{\mathrm{DR}}^{\text {pos.w.p. }}(t)=\int d \mathbf{p}_{0} \exp \left[-i \Delta S_{t}^{\epsilon}\left(\mathbf{R}, \mathbf{p}_{0}\right) / \hbar\right]\left|\psi\left(\mathbf{p}_{0}\right)\right|^{2}, \\
O_{\mathrm{DR}}^{\text {mom.w.p. }}(t)=\int d \mathbf{r}_{0} \exp \left[-i \Delta S_{t}^{\epsilon}\left(\mathbf{r}_{0}, \mathbf{P}\right) / \hbar\right]\left|\psi\left(\mathbf{r}_{0}\right)\right|^{2} .
\end{gathered}
$$

Finally, for a random mixture of pure states, i.e., an incoherent superposition of all pure basis states, the density operator as well as its Wigner function (26) is just a constant (independent of position or momenta), and for a system with a finite phase-space volume $\Omega$, the DR becomes

$$
O_{\mathrm{DR}}^{\text {rand.mixt. }}(t)=\frac{1}{\Omega} \int d \mathbf{r}_{0} \int d \mathbf{p}_{0} \exp \left(-i \Delta S_{t}^{\epsilon} / \hbar\right) .
$$

Since fidelity amplitude of a random mixture is an average fidelity amplitude of pure basis states in any basis, it is in particular equal to the average fidelity amplitude in random pure-state basis. But this has been in turn shown [14] to be equal (up to an asymptotic term $1 / n$ ) to the so-called fidelity of a random pure state (i.e., fidelity averaged over a Gaussian ensemble). So in the limit of large $n$, expression (34) can also be used to compute fidelity of a random pure state. This connection is not true for other mixed states.

It should be pointed out that while names like "position" or "momentum" states have been used to describe the special cases, they do not necessarily need to be eigenstates of the usual position or momentum operator. In the case of abstract Hilbert space with a finite basis, "position" states are simply the basis states (called computational states in the setting of quantum information, and could be, e.g., spin eigenstates), and momentum states are simply the states defined by the discrete Fourier transform of the original basis states [34]. In Ref. [34], this generalized phase-space representation is used to show that for quite a few interesting operations on computational states, the Wigner function evolves classically. In all these cases, the DR described in Secs. II-V should be applicable if a discrete Wigner function [34] is used and other simple modifications are made to account for the finitesize of phase space. In fact, this is done in the numerical examples in the following section.

\section{NUMERICAL TESTS}

Now let us apply the theoretical analysis from previous sections to a specific system, the Chirikov standard map. Its advantage is that it is discrete, coordinate space is only one dimensional, but at the same time standard map already con- tains generic complexities of classical dynamics. Specifically, the phase space is mixed and so various simplifications applicable in quasi-integrable or strongly chaotic systems are in general not applicable. Haug et al. [41] provide a detailed theoretical analysis as well as a proposal of an experiment to study quantum fidelity of the standard map.

The standard map is a symplectic map defined on a compact two-dimensional phase-space torus, as follows:

$$
\begin{gathered}
q_{j+1}=q_{j}+p_{j} \quad(\bmod 2 \pi), \\
p_{j+1}=p_{j}-\nabla W\left(q_{j+1}\right)-\epsilon \nabla V\left(q_{j+1}\right) \quad(\bmod 2 \pi),
\end{gathered}
$$

where $q$ and $p$ are position and momentum on the torus, potential $W(q)=-k \cos q$, and the perturbation is $V(q)=$ $-\cos 2 q$. Using an $n$-dimensional Hilbert space for the quantized map fixes the effective Planck constant to be $\hbar$ $=(2 \pi n)^{-1}$. Heisenberg time $t_{H} \sim n$. (We are using letter $q$ for the coordinate to distinguish this special system from the general considerations. Similarly, we will use letter $Q$ to denote the position of a position state or center of a wave packet.) The parameter $k$ controls the type of dynamics the system exhibits. For $k \ll 1$, the map is close to being integrable, for $k \gg 1$, the map is strongly chaotic. The goal of this section is not to use the dephasing representation to explore various universal regimes that occur in these two limits and have been carefully studied in the literature. This was already done in Refs. [23,42]. The goal of this section is rather to explore the detailed features of fidelity in nonuniversal regimes. The most interesting region of parameter space is in the vicinity of $k=1$, since there phase space has a significant amount of chaotic as well as integrable regions. Mixed phase space is in general the hardest to treat and therefore this setting is chosen here because it provides the most challenging test for any approximation.

\section{A. Gaussian wave packets}

One might think that general DR (21) is only useful for highly nonlocal states and that the original expression (27) from Ref. [23] is good enough at least for Gaussian wave packets. This subsection demonstrates that even for Gaussian wave packets, general DR (21) is superior to the original expression (27) from Ref. [23].

Figure 1 compares three approximations to compute fidelity of Gaussian wave packets with the exact result: (i) expression (27), originally derived in Ref. [23], for wave packets localized in position, (ii) corresponding expression (31) for wave packets localized in momentum, and (iii) the general DR (21), with the Wigner function (29), symmetrically treating position and momentum. Exact fidelity is computed by an algorithm described in Ref. [23].

For a wave packet localized in position $(\sigma=0.004 \pi)$ in Fig. 1(a), the original expression (27) from Ref. [23] works very well and is almost indistinguishable from the general DR (21), as expected, whereas Eq. (31) for momentum wave packets fails. For a wave packet localized in momentum $(\sigma$ $=0.16 \pi)$ in Fig. 1(b), the momentum-wave-packet expression (31)) works well and it is almost indistinguishable from the general DR (21), but the original position-wave-packet 

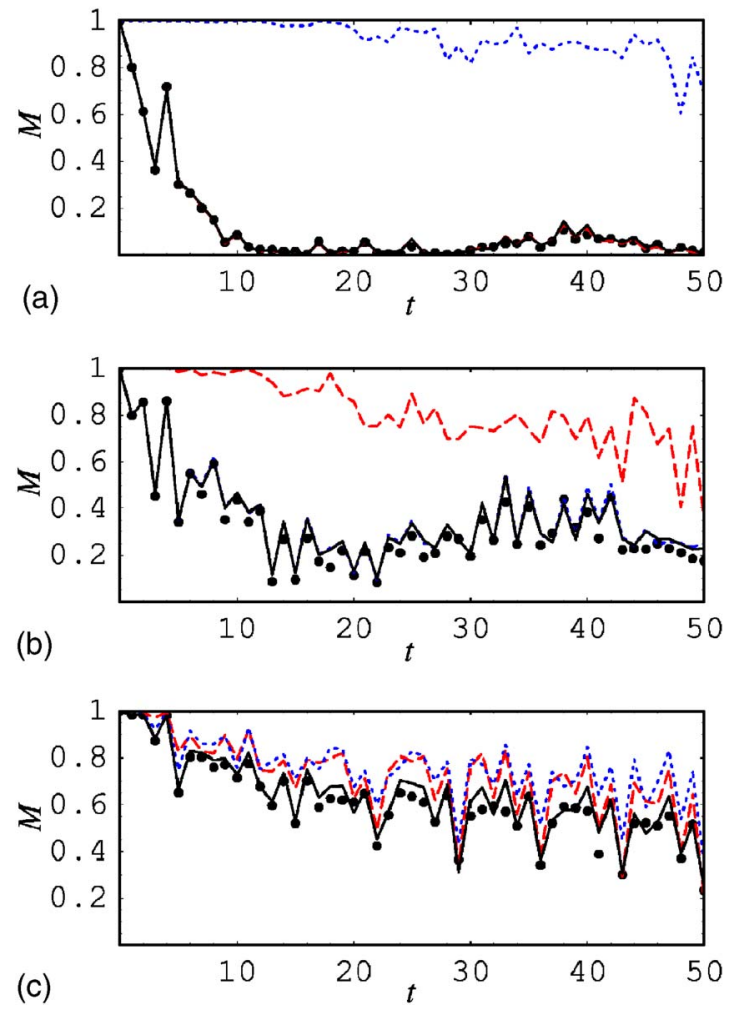

FIG. 1. (Color online) Fidelity for a Gaussian wave packet centered at $Q=0.7 \pi, P=0.4 \pi$ in a perturbed standard map with $n$ $=1000, k=0.95, \epsilon=0.015$. Comparison of the exact result (solid dots), general DR (solid line), and its specialized forms for positionlike (red-dashed line) and momentumlike wave packets (bluedotted line). The initial position spread $\sigma$ of the Gaussian is (a) $0.004 \pi$, (b) $0.16 \pi$, and (c) $0.04 \pi$. The DR calculations used 1000 classical trajectories.

expression (27) from Ref. [23] fails completely. The general DR works very well in both cases. It might seem that either the momentum or position versions could cover the whole range of Gaussian wave packets, because one might think that the intermediate case, i.e., a fairly symmetric wave packet, is localized enough in both position and momentum. That this is not so is provided by the final test in Fig. 1(c) $(\sigma=0.04 \pi)$ : both specialized expressions (30) and (31) give a significant error in comparison with exact fidelity, but the general DR (21) gives very accurate results, as expected because of its "fair" treatment of position and momentum. To conclude, expression (21), is accurate for the whole range of Gaussian wave packets, from positionlike to symmetric to momentumlike, even in the presence of mixed dynamics.

It should be noted that another "uniform" SC expression appeared in the literature [43]. It improved on the SC expression for fidelity by Vaníček and Heller [23], by expanding the action about the central trajectory to second instead of first order in position difference. For this reason, it would still work only for position localized wave packets, such as in Fig. 1(a) and would fail for cases in Figs. 1(b) and 1(c). Advantage of the dephasing representation (21), published in Ref. [27], is that it does not approximate the action: it computes the action exactly, or effectively expands the action to infinite order in the position difference.

\section{B. Nonlocal states}

For nonlocal states, there is even less hope that the position-wave-packet expression for fidelity (30) from Ref. [23] would work. One might think that for a superposition of localized wave packets it is enough to simply add the terms (30) for fidelity amplitude. This is not the case which can be seen by considering a wave packet $\psi$ that is a superposition of two Gaussian wave packets $\psi_{1}$ and $\psi_{2}$, centered at phase space points $\left(\mathbf{R}_{1}, \mathbf{P}_{1}\right)$ and $\left(\mathbf{R}_{2}, \mathbf{P}_{2}\right)$. The resulting wave packet has a Wigner function that is not just a simple sum of the Wigner functions of the two Gaussian wave packets. The correct Wigner function has in addition an interference term localized in the vicinity of the phase-space point $\left[\left(\mathbf{R}_{1}\right.\right.$ $\left.\left.+\mathbf{R}_{2}\right) / 2,\left(\mathbf{P}_{1}+\mathbf{P}_{2}\right) / 2\right]$. We will demonstrate now the importance of this interference term and show that if it is taken into account, general DR (21) will still give excellent results, even for nonlocal states.

Being motivated by the quantum computation applications, let us consider a superposition of computational states (i.e., position states in the abstract phase space), instead of Gaussian wave packets. Our initial state is a coherent superposition,

$$
|\psi\rangle=\frac{1}{\sqrt{2}}\left(\left|\mathbf{R}_{1}\right\rangle+\left|\mathbf{R}_{2}\right\rangle\right),
$$

with a Wigner distribution,

$$
\begin{aligned}
\rho_{W}^{\mathrm{coh}}(\mathbf{r}, \mathbf{p})= & \frac{1}{2}(2 \pi \hbar)^{-d}\left\{\delta\left(\mathbf{r}-\mathbf{R}_{1}\right)+\delta\left(\mathbf{r}-\mathbf{R}_{2}\right)\right. \\
& \left.+2 \delta\left[\mathbf{r}-\left(\mathbf{R}_{1}+\mathbf{R}_{2}\right) / 2\right] \cos \left[\left(\mathbf{R}_{1}-\mathbf{R}_{2}\right) \cdot \mathbf{p} / \hbar\right]\right\} .
\end{aligned}
$$

If the interference term is neglected, we obtain a Wigner function of the incoherent superposition (38),

$$
\rho_{W}^{\text {incoh }}(\mathbf{r}, \mathbf{p})=\frac{1}{2}(2 \pi \hbar)^{-d}\left[\delta\left(\mathbf{r}-\mathbf{R}_{1}\right)+\delta\left(\mathbf{r}-\mathbf{R}_{2}\right)\right] .
$$

Figure 2 compares two approximate ways to compute fidelity with the exact quantum result: both approximations use general DR (21), but whereas one uses the correct full Wigner function (36), the other uses the incorrect Wigner function (37), neglecting the interference term. The coordinate distance of the two component states varies in the two parts. If positions $\mathbf{R}_{1}$ and $\mathbf{R}_{2}$ are largely separated, oscillations in the interference term have a high frequency. Because nearby initial conditions follow similar trajectories and have similar actions, the phase factor in the DR (21) varies slowly. Therefore the fast oscillations in the weight factor given by the interference term in the Wigner function can completely cancel out the contribution of the interference part to the DR integral. (Incidentally, this situation is in a way opposite to the usual SC considerations where the weight is a slowly varying function and the phase factor is the fast oscillating factor.) Figure 2(a) shows an example of situation where this cancellation occurs: $Q_{1}=0.4 \pi$ and $Q_{2}=1.2 \pi$. Because the interference term is negligible, both approximations give the same and very accurate results. 

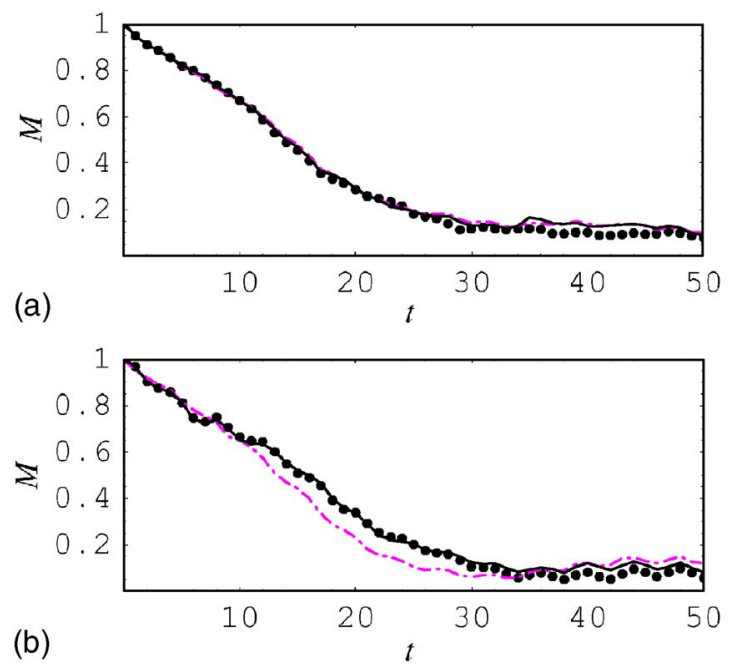

FIG. 2. (Color online) Fidelity for a nonlocal state-coherent superposition of two position states-in a perturbed standard map with $n=200, k=0.7, \epsilon=0.02$. Comparison of the exact result (solid dots), general DR (solid line), and the approximate DR neglecting coherence effects in the Wigner function (purple dashed-dotted line). The two position states are located at (a) $Q_{1}=0.4 \pi$ and $Q_{2}$ $=1.2 \pi$, (b) $Q_{1}=0.4 \pi$ and $Q_{2}=0.42 \pi$. In both parts, 400 classical trajectories were used.

If the initial states are closer, as in Fig. 2(b), where $Q_{1}$ $=0.4 \pi$ and $Q_{2}=0.42 \pi$, the interference term is important, and only the DR with the correct Wigner function (36) agrees well with the exact result. This shows that for coherent nonlocal states, general DR (21) must be used instead of some approximate versions which neglect quantum coherence of the initial state.

\section{Mixed states}

Wigner function (37) was wrong for the coherent state (35), but it does correctly describe a certain mixed state, namely the incoherent superposition of computational states $\left|\mathbf{R}_{1}\right\rangle$ and $\left|\mathbf{R}_{2}\right\rangle$,

$$
\rho^{\text {incoh }}=\frac{1}{2}\left(\left|\mathbf{R}_{1}\right\rangle\left\langle\mathbf{R}_{1}|+| \mathbf{R}_{2}\right\rangle\left\langle\mathbf{R}_{2}\right|\right) .
$$

In Sec. III it was shown that if the generalized definition (24) of fidelity for mixed states is used, dephasing representation (21) remains valid, as long as the Wigner transform of the density operator (26) is used. Figure 3 compares DR (21) with the Wigner function (37) with the exact fidelity for the mixed state (38). The agreement is again very good.

Last but not least we consider the random mixture of pure states. It is an incoherent superposition of all computational states and in a finite-dimensional Hilbert space, its density operator is

$$
\rho^{\text {rand.mixt. }}=\frac{1}{n} \sum_{i=1}^{n}\left|Q_{i}\right\rangle\left\langle Q_{i}\right|=\frac{1}{n} \hat{1} .
$$

Figure 4 compares the random-mixture version (34) of the DR with the exact result. It is reassuring that even in the case

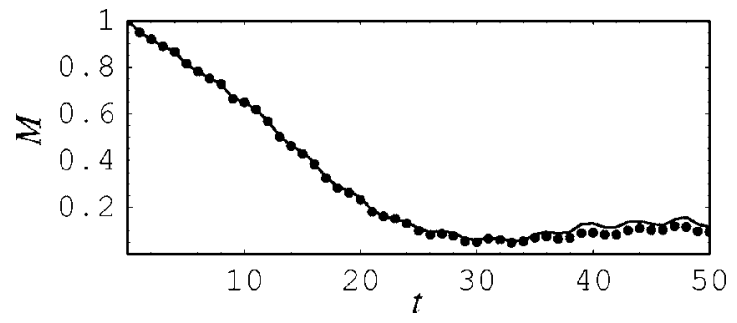

FIG. 3. Fidelity for a mixed state-incoherent superposition of two position states - in a perturbed standard map. All parameters are the same as in Fig. 2(b), except that only 200 trajectories were used. Comparison of the exact result (solid dots) and general DR (solid line).

that the whole phase space is important, with just 1000 trajectories, the DR still works so well-despite the fact that it was derived solely from SC arguments and requires only classical information.

Even though the match between the exact calculation and the dephasing representation was very good in all examples above, there was some remaining discrepancy, especially at long times. The following section therefore discusses numerical errors that are present in the computational implementation of the DR.

\section{NUMERICAL ERRORS OF THE DEPHASING REPRESENTATION}

Besides the intrinsic errors of the approximation, numerical evaluation causes additional deviations from exact fidelity amplitude. If evaluated exactly, the dephasing representation

$$
O_{\mathrm{DR}}(t)=\int d \mathbf{x} \rho_{W}(\mathbf{x}) e^{-i \Delta S_{t}^{\epsilon}(\mathbf{x}) / \hbar}=\left\langle e^{-i \varphi}\right\rangle
$$

considers all classical trajectories with their proper weight. Numerical implementation of the DR,

$$
O_{\text {num }}(t)=\frac{1}{N} \sum_{j=1}^{N} e^{-i \Delta S_{t, j}^{\epsilon} / \hbar}=\frac{1}{N} \sum_{j=1}^{N} e^{-i \varphi_{j}},
$$

computes fidelity amplitude as an average over only a finite number $N$ of trajectories. There are three types of numerical errors that affect expression (40): (i) the error of evaluating

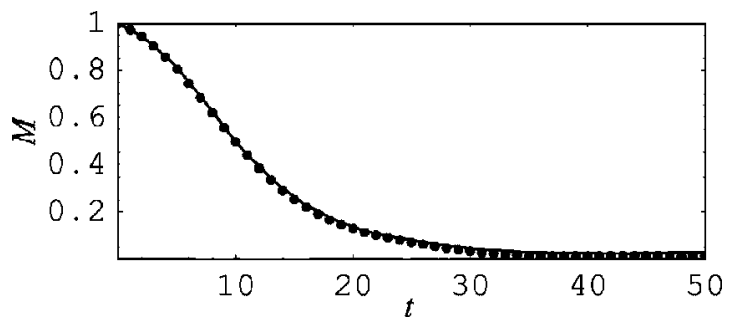

FIG. 4. Fidelity for a random mixed state-incoherent superposition of all basis states - in a perturbed standard map with $n=100$, $k=2, \epsilon=0.03$. Comparison of the exact (solid dots) result and general DR (solid line). 1000 classical trajectories were used. 
the phase $\varphi_{j}$ of each term (action difference $\Delta S_{t, j}^{\epsilon}$ ); (ii) the error due to averaging over only a finite number of terms; (iii) the error due to adding up oscillatory terms.

The first type of error, i.e., the error in fidelity amplitude due to errors in evaluating actions, can be represented by replacing the exact phase $\varphi$ by $\varphi+\Delta \varphi$ in Eq. (39),

$$
O_{\text {numl } 1}(t)=\left\langle e^{-i(\varphi+\Delta \varphi)}\right\rangle .
$$

Assuming that numerical errors in phases are uncorrelated to the phases themselves,

$$
O_{\mathrm{num} 1}(t)=\left\langle e^{-i \varphi}\right\rangle\left\langle e^{-i \Delta \varphi}\right\rangle=O_{\mathrm{DR}}(t)\left\langle e^{-i \Delta \varphi}\right\rangle .
$$

Furthermore, assuming that numerical errors at different time steps are uncorrelated, accumulation of error in phase follows a random walk, with a variance (at time $t$ )

$$
\left\langle(\Delta \varphi)^{2}\right\rangle_{t}=\delta^{2} t,
$$

where $\delta$ is the error in phase per time step. Expression (42) simplifies to

$$
O_{\text {num } 1}(t)=O_{\mathrm{DR}} e^{-\left\langle(\Delta \varphi)^{2}\right\rangle_{t} / 2}=O_{\mathrm{DR}}(t) e^{-\delta^{2} t / 2} .
$$

If the only numerical error were in the phases of various terms, numerical fidelity would be smaller than the exact value $O_{\mathrm{DR}}$ by a factor $e^{-\delta^{2} t / 2}$.

The second and most important type of error is due to averaging over a finite number of terms,

$$
O_{\text {num2 }}(t)=\frac{1}{N} \sum_{j=1}^{N} e^{-i \varphi_{j}} .
$$

Let $P(\varphi)$ denote the distribution function of phases. It satisfies the normalization condition

$$
\int P(\varphi) d \varphi=1
$$

In general, there could be two types of error of fidelity amplitude, systematic and statistical,

$$
\begin{gathered}
\sigma_{\text {syst }}=\left\langle O_{\text {num }}\right\rangle-O_{\mathrm{DR}}, \\
\sigma_{\text {stat }}=\sqrt{\left\langle\left|O_{\text {num }}-\left\langle O_{\text {num }}\right\rangle\right|^{2}\right\rangle}=\sqrt{\left\langle\left|O_{\text {num }}\right|^{2}\right\rangle-\left|\left\langle O_{\text {num }}\right\rangle\right|^{2}} .
\end{gathered}
$$

The brackets $\langle\cdots\rangle$ denote the average over the distribution $P(\varphi)$. The systematic error vanishes for fidelity amplitude, $\sigma_{\text {syst }}=0$, since

$$
\begin{gathered}
O_{\mathrm{DR}}(t)=\int P(\varphi) e^{-i \varphi} d \varphi, \\
\left\langle O_{\mathrm{num}}(t)\right\rangle=\frac{1}{N} \int d \varphi_{1} P\left(\varphi_{1}\right) \cdots \int d \varphi_{N} P\left(\varphi_{N}\right) \sum_{j=1}^{N} e^{-i \varphi_{j}} \\
=\frac{1}{N} \sum_{j=1}^{N} O_{\mathrm{DR}}(t)=O_{\mathrm{DR}}(t) .
\end{gathered}
$$

The statistical error is nonzero because

$$
\begin{aligned}
\left\langle\left|O_{\text {num }}(t)\right|^{2}\right\rangle & =\frac{1}{N^{2}} \int d \varphi_{1} P\left(\varphi_{1}\right) \cdots \int d \varphi_{N} P\left(\varphi_{N}\right)\left|\sum_{j=1}^{N} e^{-i \varphi_{j}}\right|^{2} \\
& =\frac{1}{N^{2}}\left[N+N(N-1)\left|O_{\mathrm{DR}}(t)\right|^{2}\right] \\
& =\frac{1}{N}+\left(1-\frac{1}{N}\right)\left|O_{\mathrm{DR}}(t)\right|^{2} .
\end{aligned}
$$

It follows from Eqs. (46)-(48) that the statistical error squared is

$$
\sigma_{\text {stat }}^{2}=\frac{1}{N}\left[1-\left|O_{\mathrm{DR}}(t)\right|^{2}\right]
$$

For small values of fidelity (i.e., in general for long times or large perturbations), the statistical error is approximately $1 / \sqrt{N}$. For short times, when $\left|O_{\mathrm{DR}}(t)\right| \approx 1$, the statistical error vanishes. A similar analysis can be performed for fidelity itself. In fact this is the quantity that is displayed in Figs. $1-4$. For fidelity, the systematic error is nonzero because

$$
\sigma_{\text {syst }}=\left\langle\left|O_{\text {num }}\right|^{2}\right\rangle-\left|O_{\mathrm{DR}}(t)\right|^{2}=\frac{1}{N}\left[1-\left|O_{\mathrm{DR}}(t)\right|^{2}\right] .
$$

The statistical error squared is

$$
\sigma_{\text {stat }}^{2}=\left\langle\left(\left|O_{\text {num }}\right|^{2}-\left\langle\left|O_{\text {num }}\right|^{2}\right\rangle\right)^{2}\right\rangle=\left\langle\left|O_{\text {num }}\right|^{4}\right\rangle-\left\langle\left|O_{\text {num }}\right|^{2}\right\rangle^{2} .
$$

It is a bit harder to evaluate since $\left\langle\left|O_{\text {num }}\right|^{4}\right\rangle$ has several types of "cross terms," but following a procedure similar to Eq. (48), the statistical error squared (to leading order in inverse powers of $N$ ) is

$$
\begin{aligned}
\sigma_{\text {stat }}^{2}= & \frac{2}{N}\left[\operatorname{Re}\left(\left\langle e^{2 i \varphi}\right\rangle\left\langle e^{-i \varphi}\right\rangle^{2}\right)+\left|O_{\mathrm{DR}}(t)\right|^{2}-2\left|O_{\mathrm{DR}}(t)\right|^{4}\right] \\
& +O\left(1 / N^{2}\right) .
\end{aligned}
$$

For small values of fidelity, statistical error will be of the order $O\left[\left|O_{\mathrm{DR}}(t)\right| / \sqrt{N}\right]$. For short times or small perturbations when $\left|O_{\mathrm{DR}}(t)\right| \approx 1$, the statistical error will again vanish since the three terms in the square brackets will exactly cancel each other.

The third type of error is due to adding up oscillatory terms. It can be represented as

$$
O_{\text {num3 }}(t)=\frac{1}{N} \sum_{j=1}^{N}\left(1+\delta_{j}\right) e^{-i \varphi_{j}},
$$

i.e., each term has the correct phase, but a slightly perturbed amplitude. Simplifying expression (53) assuming that numerical errors $\varphi_{j}$ are uncorrelated with phases $\varphi_{j}$,

$$
O_{\text {num } 3}(t)=O_{\text {num } 2}(t)+\frac{1}{N} \sum_{j=1}^{N} \delta_{j} e^{-i \varphi_{j}}
$$




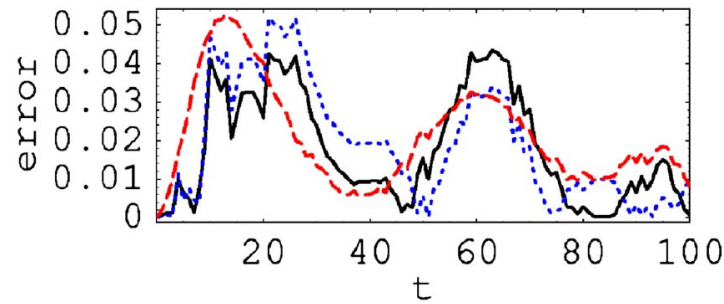

FIG. 5. (Color online) Dependence of numerical errors of DR on time for the random mixture with the same parameters as in Fig. 4, except $N=100$ : $\left|M_{\text {num }}-M_{\mathrm{QM}}\right|$ (solid line), $\left|\tilde{M}_{\text {num }}-M_{\mathrm{QM}}\right|$ (bluedotted line), and $\sigma_{\text {stat }}$ (red-dashed line).

$$
\begin{aligned}
& =O_{\text {num2 }}(t)+\frac{1}{N} \sum_{j=1}^{N} \delta_{j} \frac{1}{N} \sum_{k=1}^{N} e^{-i \varphi_{k}} \\
& =O_{\text {num } 2}(t)\left(1+\frac{1}{N} \sum_{j=1}^{N} \delta_{j}\right) .
\end{aligned}
$$

Assuming that errors of different terms are uncorrelated, the effect of these errors on $O_{\text {num }}$ can be described as

$$
O_{\mathrm{num} 3}(t) \approx O_{\mathrm{DR}}(t)(1 \pm \delta / \sqrt{N}),
$$

where $\delta$ is the magnitude of error in amplitude of a typical term. The relative error in $O_{\text {num } 3}(t)$ is proportional to the accuracy $\delta$ and inversely proportional to the square root of the number of trajectories.

Errors of $O_{\text {num } 1}(t)$ and $O_{\text {num3 }}(t)$ depend on the accuracy of calculation and can be diminished by improving this numerical accuracy. The second type of error, of $O_{\text {num2 }}(t)$ depends only on the exact (non-numerical) averages and on the number $N$ of classical trajectories used. Therefore this appears to be the most important source of error in the numerical calculation of $O_{\mathrm{DR}}$. In the case of fidelity rather than fidelity amplitude, the nonzero systematic error (50) for finite $N$ suggests that the numerical result could be improved by replacing $M_{\text {num }}=\left|O_{\text {num }}\right|^{2}$ by

$$
\begin{aligned}
\tilde{M}_{\text {num }} & =\left|O_{\text {num }}\right|^{2}-\sigma_{\text {syst }}=\left|O_{\text {num }}\right|^{2}-\frac{1}{N}\left(1-\left|O_{\mathrm{DR}}\right|^{2}\right) \\
& \approx\left|O_{\text {num }}\right|^{2}\left(1+\frac{1}{N}\right)-\frac{1}{N} .
\end{aligned}
$$

Figure 5 compares three types of errors: $\left|M_{\text {num }}-M_{\mathrm{QM}}\right|$, $\left|\tilde{M}_{\text {num }}-M_{\mathrm{QM}}\right|$, and $\sigma_{\text {stat }}$ according to Eq. (52) for a random mixture from Fig. 4. Already for $N=100$ that is used in the figure, the systematic error (50) is quite small, so $\mid M_{\text {num }}$ $-M_{\mathrm{QM}} \mid$ is very close to $\left|\tilde{M}_{\text {num }}-M_{\mathrm{OM}}\right|$. Both numerical errors also follow very closely statistical error $\sigma_{\text {stat }}$ computed according to the theoretical estimate (52).

Figure 6 shows the dependence of three types of timeaveraged errors on the number $N$ of classical trajectories used: the time-averaged numerical error $\left[(1 / t) \sum_{\tau=1}^{t}\left|M_{\text {num }}(\tau)-M_{\mathrm{QM}}(\tau)\right|^{2}\right]^{1 / 2}$, an analogous time average for $\tilde{M}_{\text {num }}$, and the time-averaged statistical error $\left[(1 / t) \sum_{\tau=1}^{t} \sigma_{\text {stat }}^{2}(\tau)\right]^{1 / 2}$. As expected, the numerical error fol-

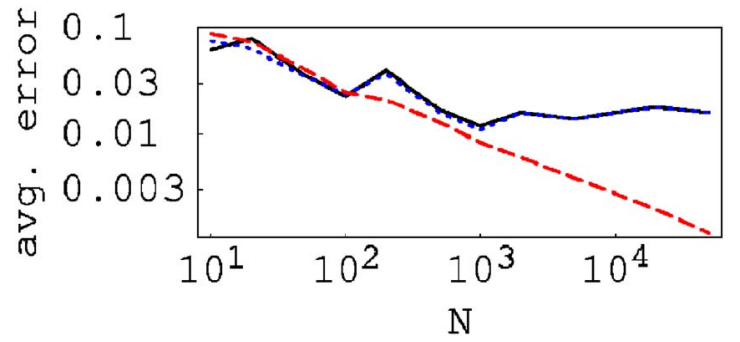

FIG. 6. (Color online) Dependence of time-averaged numerical errors $\left(\left[(1 / t) \sum_{\tau=1}^{t} \sigma^{2}(\tau)\right]^{1 / 2}\right)$ of DR on the number $N$ of classical trajectories: $\sigma=\left|M_{\text {num }}-M_{\mathrm{QM}}\right|$ (solid line), $\sigma=\left|\tilde{M}_{\text {num }}-M_{\mathrm{QM}}\right|$ (bluedotted line), and $\sigma=\sigma_{\text {stat }}$ (red-dashed line). Parameters are the same as in Fig. 5, in particular $t=100$.

lows the statistical error for smaller values of $N$ (approximately up to $N \approx n$ ). For larger values of $N$, the statistical error decreases further, but the numerical error saturates at a horizontal asymptote due to the intrinsic error of the dephasing representation.

\section{RELATION TO OTHER “WIGNER” METHODS}

It should be noted that the Wigner distribution has been used in various other approximate methods, especially in chemical physics. For instance, it was used to compute photodissociation cross sections $[44,45]$, to treat inelastic scattering [46], or to compute thermal correlation functions using the linearized SC IVR method $[25,47,48]$. In all these applications, there was just one Hamiltonian, but the two states (or more generally, density or other operators) were different. The quantity of interest was a general correlation function of the type

$$
C_{A B}(t)=\operatorname{tr}\left(A U^{\dagger} B U\right)
$$

where $A$ and $B$ are general operators and $U=\mathcal{T} e^{-i \int H d \tau}$ is the time evolution operator. Using various approximations, all authors [25,44-48] obtain the same final result, expressed as an overlap of two Wigner distributions, one at time 0 , the other evolved classically to time $t$,

$$
C_{A B}^{\mathrm{Wigner}}(t)=(2 \pi \hbar)^{d} \int d \mathbf{r}_{0} \int d \mathbf{p}_{0} A_{W}\left(\mathbf{r}_{0}, \mathbf{p}_{0}\right) B_{W}\left(\mathbf{r}_{t}, \mathbf{p}_{t}\right) .
$$

Here $A_{W}$ and $B_{W}$ are the Wigner transforms (26) of operators $A$ and $B$.

Because there is only one Hamiltonian, there is no dephasing factor $e^{i \Delta S / \hbar}$, as in the DR. In fact we could apply one of these older approaches to the second generalized definition (25) of fidelity for mixed states because that definition is in the form of Eq. (54) with $A=B=\rho$ and the time evolution operator $U=e^{+i H^{\epsilon} t / \hbar} e^{-i H^{0} t / \hbar}$. Then we would obtain a very different result from the DR, 


$$
M^{\text {Wigner }}(t)=(2 \pi \hbar)^{d} \int d \mathbf{r}_{0} \int d \mathbf{p}_{0} \rho_{W}\left(\mathbf{r}_{0}, \mathbf{p}_{0}\right) \rho_{W}\left(\mathbf{r}_{t}, \mathbf{p}_{t}\right)
$$

Although appearing as elegant as the DR, there is a problem with this expression. First, it will be much more sensitive to numerical errors. We can see that already by considering zero perturbation. Correctly, for each initial condition $\mathbf{r}_{0}, \mathbf{p}_{0}$, we should have $\mathbf{r}_{0}=\mathbf{r}_{t}$ and $\mathbf{p}_{t}=\mathbf{p}_{0}$. In systems with nonlinear dynamics, particularly chaotic systems, numerical errors in forward and backward propagation will yield exponentially growing errors. If the initial state is a localized wave packet, expression (56) would give a numerically decaying overlap even for zero perturbations when exact fidelity is constant $M(t)=1$. Indeed, a numerical test not presented here showed that instead of staying at unity forever, $M^{\text {Wigner }} \approx 1$ for a finite time and then decays exponentially.

Even if numerical errors did not exist, Eq. (56) would have problems. It can describe some interference, but only that due to the oscillating parts in the Wigner function of the initial state. For simple Gaussian wave packets, the Wigner function is positive and the fidelity decay in Eq. (56) is completely due to the decay of classical overlaps, i.e., classical fidelity. To conclude, the "Wigner" form (56) is apparently not as good as the DR because it does not account for "dynamical" dephasing, but it does deserve further study, especially because it might shed further light on the question of the importance of various contributions to fidelity. Numerical tests not presented here show that, as expected, $M^{\text {Wigner }}$ correctly describes exact fidelity in both chaotic and quasiintegrable systems for large perturbations (i.e., in Lyapunov and algebraic regimes, respectively), when the decay can be described classically [42]. It gives wrong results in both chaotic and quasi-integrable systems for small perturbations (in the FGR and Gaussian regimes), when the decay cannot be described classically [42].

\section{CONCLUSION}

This paper has presented a derivation of a general semiclassical expression for fidelity of pure and mixed states. This dephasing representation expresses fidelity as an interference integral, with weight of each term given by the Wigner function of the initial state and the phase by the integrated perturbation along an unperturbed trajectory. In particular, no analog of the Van Vleck determinant is needed. As the original specialized expression (30) from Ref. [23], the DR avoids searching for the exponentially growing number of terms in the standard SC expressions [4]. It also avoids the ubiquitous divergences in Van Vleck determinants present in the usual SC expressions.
The justification of the DR provides an interesting application of the shadowing theorem. Instead of validating the numerically noisy calculation of classical averages, here shadowing was used to support a SC computation of a quantum object-quantum fidelity. Another aspect that makes the DR a "bridge" between classical and quantum mechanics is the following: On one hand, similarly as Van Vleck's propagator, but unlike Feynman's path integral, the DR uses only classically allowed trajectories. On the other hand, similarly as Feynman's path integral, but unlike Van Vleck's propagator, the DR gives each trajectory the same dynamical weight (the only weight-given by the Wigner function-is due to the initial condition).

The advantage of DR lies in that it does not require the original state to be localized. Its form suggests that it should be applicable to general pure and mixed states. This claim was supported by the following numerical evidence: First, it was shown, in the example of Gaussian wave packets, that position and momentum must be treated symmetrically. This was the shortcoming of the expression from Ref. [23] and is corrected in the DR. Second, in the example of coherent superpositions of states, it was shown that oscillatory patterns in the Wigner function are important. This is related to the issue of importance of sub-Planck structures on decoherence $[17,49]$. Finally, it was shown that the DR is also accurate for mixed states: incoherent superpositions and random mixtures.

While the numerical tests were quite successful, the DR breaks down in the perturbative regime after Heisenberg time [23]. This should not be discouraging since then the discrete features of the spectrum become important. On the other hand, dephasing representation covers six other known universal regimes (Fermi Golden Rule [4,6,7,10], Lyapunov [4], Gaussian [10], algebraic [12], superexponential [13] regimes, and fidelity freeze [15]) as well as nonuniversal regimes of fidelity decay for generic pure and mixed states. Since the dephasing representation (21) appears to be much closer to the exact formulation of fidelity in the interaction picture [11] than the starting SC approximation, a more rigorous estimate of the errors should be attainable.

Finally, the DR appears to be the method of choice for approximate but accurate calculation of fidelity in nonuniversal regimes in many-dimensional systems due to the ease of its computational implementation in comparison with exact quantum methods.

\section{ACKNOWLEDGMENTS}

The author wishes to thank the Department of Chemistry at the University of California, Berkeley, and the Institute for Advanced Study for support. 
[1] A. Peres, Phys. Rev. A 30, 1610 (1984).

[2] M. A. Nielsen and I. L. Chuang, Quantum Computation and Quantum Information (Cambridge University Press, Cambridge, England, 2000).

[3] T. Prosen, T. H. Seligman, and M. Žnidarič, Prog. Theor. Phys. Suppl. 150, 200 (2003).

[4] R. A. Jalabert and H. M. Pastawski, Phys. Rev. Lett. 86, 2490 (2001).

[5] F. M. Cucchietti, H. M. Pastawski, and R. A. Jalabert, Phys. Rev. B 70, 035311 (2004).

[6] P. Jacquod, P. G. Silvestrov, and C. W. J. Beenakker, Phys. Rev. E 64, 055203(R) (2001).

[7] N. R. Cerruti and S. Tomsovic, Phys. Rev. Lett. 88, 054103 (2002).

[8] N. R. Cerruti and S. Tomsovic, J. Phys. A 36, 3451 (2003).

[9] N. R. Cerruti and S. Tomsovic, J. Phys. A 36, 11915 (2003).

[10] T. Prosen, Phys. Rev. E 65, 036208 (2002).

[11] T. Prosen and M. Žnidarič, J. Phys. A 35, 1455 (2002).

[12] P. Jacquod, I. Adagidelli, and C. W. J. Beenakker, Europhys. Lett. 61, 729 (2003).

[13] P. G. Silvestrov, J. Tworzydlo, and C. W. J. Beenakker, Phys. Rev. E 67, 025204(R) (2003).

[14] T. Prosen and M. Žnidarič, New J. Phys. 5, 109 (2003).

[15] T. Prosen and M. Žnidarič, Phys. Rev. Lett. 94, 044101 (2005)

[16] D. V. Bevilaqua and E. J. Heller, nlin.CD/0409007.

[17] W. H. Zurek, Nature (London) 412, 712 (2001).

[18] F. M. Cucchietti, D. A. R. Dalvit, J. P. Paz, and W. H. Zurek, Phys. Rev. Lett. 91, 210403 (2003).

[19] T. Gorin, T. Prosen, and T. H. Seligman, New J. Phys. 6, 20 (2004).

[20] H. M. Pastawski, P. R. Levstein, and G. Usaj, Phys. Rev. Lett. 75, 4310 (1995).

[21] G. Usaj, H. M. Pastawski, and P. R. Levstein, Mol. Phys. 95, 1229 (1998).

[22] R. Schafer, H.-J. Stockmann, T. Gorin, and T. H. Seligman, Phys. Rev. Lett. 95, 184102 (2005).
[23] J. Vaníček and E. J. Heller, Phys. Rev. E 68, 056208 (2003).

[24] W. H. Miller, J. Chem. Phys. 53, 3578 (1970).

[25] W. H. Miller, J. Phys. Chem. 105, 2942 (2001).

[26] W. Wang, G. Casati, B. Li, and T. Prosen, Phys. Rev. E 71, 037202 (2005)

[27] J. Vaníček, Phys. Rev. E 70, 055201(R) (2004).

[28] S. M. Hammel, J. A. Yorke, and C. Grebogi, J. Complex. 3, 136 (1987).

[29] C. Grebogi, S. M. Hammel, J. A. Yorke, and T. Sauer, Phys. Rev. Lett. 65, 1527 (1990).

[30] E. P. Wigner, Z. Phys. Chem. Abt. B 19, 203 (1932).

[31] J. H. Hannay and M. V. Berry, Physica D 1, 267 (1980).

[32] W. K. Wooters, Ann. Phys. (N.Y.) 176, 1 (1987).

[33] U. Leonhardt, Phys. Rev. Lett. 74, 4101 (1995).

[34] C. Miquel, J. P. Paz, and M. Saraceno, Phys. Rev. A 65, 062309 (2002).

[35] J. H. V. Vleck, Proc. Natl. Acad. Sci. U.S.A. 14, 178 (1928).

[36] M. C. Gutzwiller, Chaos in Classical and Quantum Mechanics (Springer-Verlag, New York, 1990).

[37] M. Sieber and K. Richter, Phys. Scr. T90, 128 (2001).

[38] P. A. Braun, F. Haake, and S. Heusler, J. Phys. A 35, 1381 (2002).

[39] D. V. Anosov, Proc. Steklov Inst. Math. 90, 1 (1967).

[40] R. Bowen, J. Diff. Eqns. 18, 333 (1987).

[41] F. Haug, M. Bienert, W. P. Schleich, T. H. Seligman, and M. G. Raizen, Phys. Rev. A 71, 043803 (2005).

[42] J. Vaníček, quant-ph/0410205.

[43] W. Wang and B. Li, Phys. Rev. E 71, 066203 (2005).

[44] E. J. Heller, J. Chem. Phys. 65, 1289 (1976).

[45] R. C. Brown and E. J. Heller, J. Chem. Phys. 75, 186 (1981).

[46] H. W. Lee and M. O. Scully, J. Chem. Phys. 73, 2238 (1980).

[47] W. H. Miller, J. Chem. Phys. 61, 1823 (1974).

[48] H. Wang, X. Sun, and W. H. Miller, J. Chem. Phys. 108, 9726 (1998).

[49] P. Jacquod, I. Adagideli, and C. W. J. Beenakker, Phys. Rev. Lett. 89, 154103 (2002). 